\title{
Combination of negative pressure wound therapy with open bone grafting for bone and soft tissue defects
}

\author{
KAI DENG, AI-XI YU, CHENG-YAN XIA, ZONG-HUAN LI and WEI-YANG WANG \\ Department of Micro-Orthopedics, Zhongnan Hospital of Wuhan University, Wuhan, Hubei 430071, P.R. China
}

Received January 31, 2013; Accepted June 11, 2013

DOI: $10.3892 / \mathrm{mmr} .2013 .1536$

\begin{abstract}
The aim of this study was to investigate the efficiency of negative pressure wound therapy (NPWT) combined with open bone graft (OBG; NPWT-OBG) for the treatment of bone and soft tissue defects with polluted wounds in an animal model. All rabbits with bone and soft tissue defects and polluted wounds were randomly divided into two groups, the experimental group (NPWT with bone graft) and the control group (OBG). The efficacy of the treatment was assessed by the wound conditions and healing time. Bacterial bioburdens and bony calluses were evaluated by bacteria counting and X-rays, respectively. Furthermore, granulation tissue samples from the wounds on days $0,3,7$ and 14 of healing were evaluated for blood vessels and vascular endothelial growth factor (VEGF) levels. Wounds in the experimental group tended to have a shorter healing time, healthier wound conditions, lower bacterial bioburden, improvement of the bony calluses and an increased blood supply compared with those in the control group. With NPWT, wound infection was effectively controlled. For wounds with osseous and soft tissue defects, NPWT combined with bone grafting was demonstrated to be more effective than an OBG.
\end{abstract}

\section{Introduction}

The treatment of composite bone and soft tissue defects continues to challenge orthopedic surgeons (1). Traditionally, tissue flaps and open bone grafts (OBGs) were commonly used to restore structure and function. Composite tissue flap transplantation may minimize the injury, but it is complex and unpopular (2). Conventional installment therapy does not have an extended healing time and has poor efficacy. Novel methods for the treatment of bone and soft tissue defects are required.

Correspondence to: Professor Ai-Xi Yu, Department of Micro-Orthopedics, Zhongnan Hospital of Wuhan University, 169 Donghu Road, Wuhan, Hubei 430071, P.R. China

E-mail: yuaixi666@163.com

Key words: negative pressure wound therapy, bone graft, osseous/soft tissue defect, polluted wound
OBG is a procedure that replaces the missing bone in order to repair the bone defects (3). OBG is feasible due to the fact that bone tissue has the ability to completely regenerate. OBG has been reported to significantly shorten hospital stays (4). The ensuing dressing changes did not efficiently control infections, particularly for free bone fragments without a blood supply; therefore, healing time for the wound was prolonged. This increases the risk of infection and chronic osteomyelitis. Negative pressure wound therapy (NPWT) has been widely used on various complex wounds (5). NPWT reduces local edema and bacterial load, promotes the growth of granulation tissue and increases blood supply topically, and thus promotes wound healing (6). We hypothesized that NPWT combined with OBG (NPWT-OBG) may be a novel and effective method for the management of bone and soft tissue defects.

Animal models with polluted wounds and bone and soft tissue defects were treated by either NPWT-OBG or OBG. The efficiency was assessed by bacteria counting, X-ray and evaluation of the levels of CD34 and vascular endothelial growth factor (VEGF).

\section{Materials and methods}

Animal model. In the present study, 24 New Zealand rabbits weighing 2.8-3.5 kg were purchased and housed in an approved animal care facility (eligibility certification: No. 4210000313). The rabbits were anesthetized with a $1 \%$ pentobarbital sodium $(30 \mathrm{mg} / \mathrm{kg})$ intraperitoneal injection. The forearm hair of all the rabbits was removed using $8 \% \mathrm{NaS}$ solution prior to each surgical procedure. An incision, $2.5 \mathrm{~cm}$ in length including skin and subcutaneous tissue, was made in the radialis side of each forearm. The radius was partially amputated by removing $1 \mathrm{~cm}$. A sufficient quantity of autologous ilium was obtained and grafted into the defects. The wounds were daubed with Staphylococcus aureus $\left(1 \times 10^{3}\right)$ to produce polluted wounds. After $1 \mathrm{~h}$, the polluted wounds were debrided and a bacteria counting test was performed in order to determine whether the model was successful. We randomly selected two forearms to treat with OBG or NPWT-OBG. Rabbits in the NPWT-OBG group were then treated with VAC foam (VSD Medical Science Technology Co., Ltd., Wuhan, China). The negative pressure value was $-75 \mathrm{mmHg}$. Wounds in the OBG group were covered with conventional gauze. The day on which the surgery was performed was defined as day 0 . Vacuum sealing drainage (VSD) dressing change was 
performed on days 3, 7 and 14 and granulation tissue, with a volume of $2 \times 5 \times 10 \mathrm{~mm}^{3}$, was obtained under aseptic conditions and divided into three. The samples were immediately analyzed by bacteria counting, stored at $-80^{\circ} \mathrm{C}$ for western blot analysis and immersed in $4 \%$ paraformaldehyde for immunohistochemical analysis, respectively. From day 21, the gauze dressings were changed every three days.

Bacteria counting. Samples were weighed immediately, minced, homogenized and diluted. Diluents $(5 \mu \mathrm{l})$ were placed on a normal agar plate and incubated at $37^{\circ} \mathrm{C}$ with $5 \% \mathrm{CO}_{2}$ for $48 \mathrm{~h}$. The number of bacteria in each wound was calculated by counting the colony forming units (CFUs) on each plate.

$X$-ray imaging. The lateral films for the upper extremities were performed for each rabbit on days $0,7,14,21$ and 28 . The condition of the fracture and its healing rate were recorded on the 28th day.

Immunohistochemical analysis. All samples fixed in 4\% paraformaldehyde were embedded in paraffin and routinely sectioned $(5 \mu \mathrm{m})$. Staining was performed using the SABC method (7). The primary monoclonal anti-rabbit CD34 antibody (Santa Cruz Biotechnology, Inc., Santa Cruz, CA, USA; 1:1000) was applied to the sections and incubated for $1 \mathrm{~h}$ at room temperature, rinsed three times with PBS and the sections were then incubated with fluorescein isothiocyanate (FITC)- or rhodamine-conjugated secondary antibody (Santa Cruz Biotechnology, Inc.) for $30 \mathrm{~min}$. The complex was visualized using the avidin-biotinylated enzyme complex. The stained blood microvessels were counted in the section.

Western blot analysis. All samples were homogenized in buffer with an added protease inhibitor cocktail (Roche Inc., Basel, Switzerland), $10 \mathrm{mM} \mathrm{NaCl}, 1 \% \mathrm{NP} 40,0.02 \%$ sodium azide and $50 \mathrm{mM}$ Tris. Homogenates were centrifuged at 8,465 x g for $10 \mathrm{~min}$ at $4^{\circ} \mathrm{C}$. The supernatant was stored at $-20^{\circ} \mathrm{C}$ prior to use. The loading sample volume was $50 \mu \mathrm{g}$ and the protein was separated using $10 \%$ sodium dodecyl sulfate-polyacrylamide gel electrophoresis (SDS-PAGE) and transferred to a polyvinylidene difluoride (PVDF) membrane. The membranes were blocked with $5 \%$ fat milk at $37^{\circ} \mathrm{C}$ for $2 \mathrm{~h}$ and incubated in a shaker (WD-9405A, Beijing World Biomedical Instruments Co., Ltd., Beijing, China). for a further $2 \mathrm{~h}$. The membranes were incubated with a primary goat anti-rabbit antibody against either VEGF (Santa Cruz Biotechnology, Inc.; 1:20,000) or $\beta$-actin (Santa Cruz Biotechnology, Inc.; $1: 10,000)$ overnight at $4^{\circ} \mathrm{C}$. Blots were washed for 5-10 min with Tris-buffered saline containing $0.05 \%$ Tween-20 (TBST). Following washing, the blots were incubated for $1 \mathrm{~h}$ with a horseradish-peroxidase (HRP)-coupled secondary antibody and subsequently washed again with TBST. The blots were incubated with a luminescent HRP substrate for 1 min until positive signals were detected. The optical densities of at least three replicates of each sample were quantified. Samples were stored at $-80^{\circ} \mathrm{C}$ prior to use.

Statistical analysis. All data are presented as the mean \pm SD, and were analyzed by the Student's t-test. Enumeration data are presented as percentages and were analyzed by the Chi-square test. $\mathrm{P}<0.05$ was considered to indicate a statistically significant difference.

\section{Results}

Wound condition. On days 3, 7, 14 and 21 following the surgical procedure, the wounds in the NPWT-OBG group were cleaner and drier with less exudate compared with the control group (Fig. 1). In general, the quality of granulation tissue was improved following NPWT; the wound healing rate significantly increased and the wound healing time decreased (18.5 \pm 9.4 for the NPWT-OBG group vs. 24.2 \pm 6.5 days for the control group).

Bacterial colony counts. The tissues obtained from the wounds were cultured and analyzed $1 \mathrm{~h}$ after the surgical procedure. Staphylococcus aureus, with a density $<1 \times 10^{5} \mathrm{CFU} / \mathrm{ml}$, was detected in all wounds. This suggested that the polluted wound model had been successfully established. Staphylococcus aureus was also detected on days 3 and 7 in both groups; however, the difference was not identified to be statistically significant (Fig. 2). On days 14 and 21 , no bacteria were detected. Therefore, the bacteria count was not performed on day 28 .

VEGF expression. VEGF has been confirmed to promote angiogenesis during the wound healing process (8). Overexpression of VEGF was observed in wounds treated by NPWT. In this study, in order to examine the exact mechanism underlying the effect of VEGF on wound healing, western blot analysis of VEGF was performed. At the examined times, the expression of VEGF was higher in the NPWT-OBG group than in the OBG group. The results indicated that VEGF overexpression contributed to the wound healing process.

Microvascular density. On days 3, 7 and 14, the microvessels of the wounds were counted via immunohistochemical staining of CD34 (Fig. 4). The results demonstrated that the densities of microvessels in the NPWT-OBG group were higher than those in the OBG group throughout the entire process. Moreover, the density increased as the experiment proceeded, and the difference was statistically significant $(\mathrm{P}<0.05)$.

$X$-ray examination. For all rabbits, the fracture healing processes were monitored by X-ray examination on days 0,7 , 14, 21 and 28, respectively (Fig. 5). The films revealed that the callus growth in the experimental group had improved to a greater extent than that in the control group. On the 28th day, the healing rate of the NPWT-OBG group was $75 \%(9 / 12)$, which was higher than the OBG group [25\% (3/12)].

\section{Discussion}

The pivotal issue when repairing composite bone and soft tissue defects is to restore the outline and function of the bone and soft tissue as soon as possible (1). Composite tissue flap transplantation achieves this goal in one stage. Unfortunately, the surgery is unpopular. Furthermore, donor-site problems cause pain in the patients. 

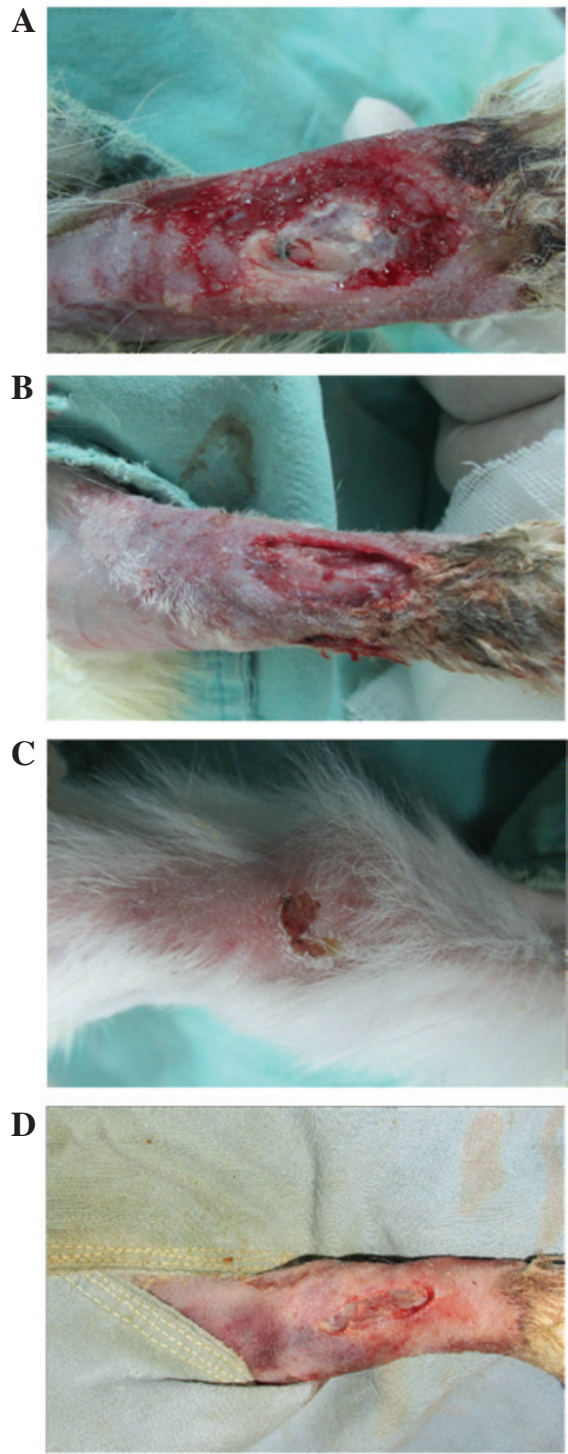

Figure 1. General conditions of the wound healing process. (A and C) Wounds on days 3 and 14, respectively, following treatment with NPWT-OBG. (B and D) Wounds on days 3 and 14, respectively, in the OBG group. NPWT-OBG, negative pressure wound therapy-open bone graft.
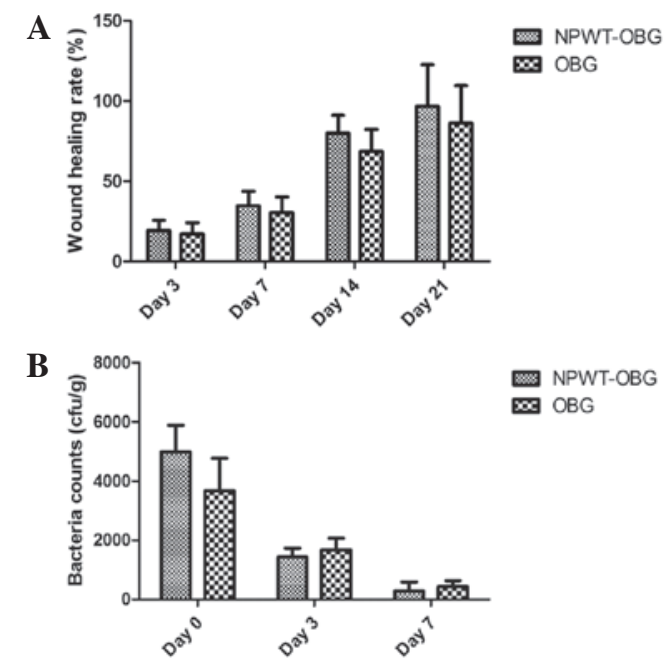

Figure 2. Comparison of (A) the wound healing rate and (B) bacteria counting at different times. NPWT, negative pressure wound therapy; OBG, open bone graft.

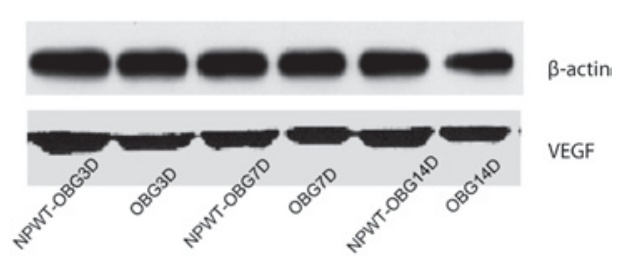

Figure 3. Western blot analysis of vascular endothelial growth factor (VEGF). $\mathrm{D}$, day; OBG, open bone graft; NPWT, negative pressure wound therapy.

A

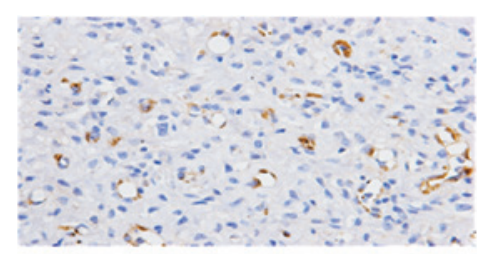

B

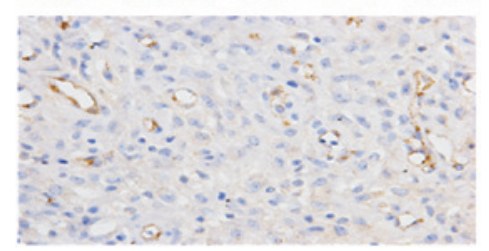

C pescens:

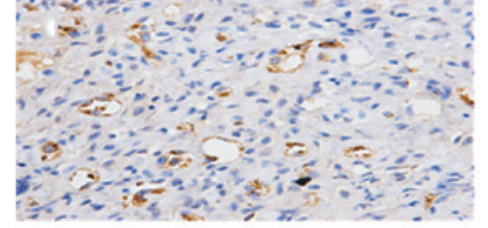

D

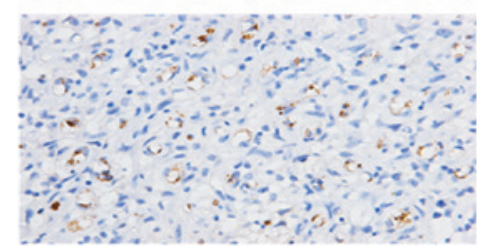

E

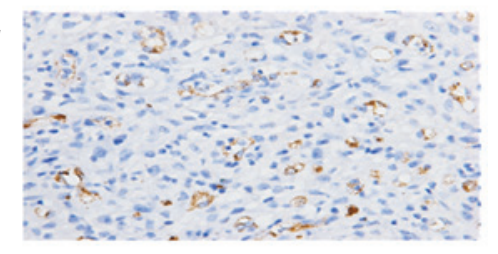

F

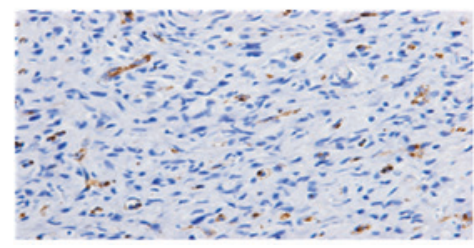

G

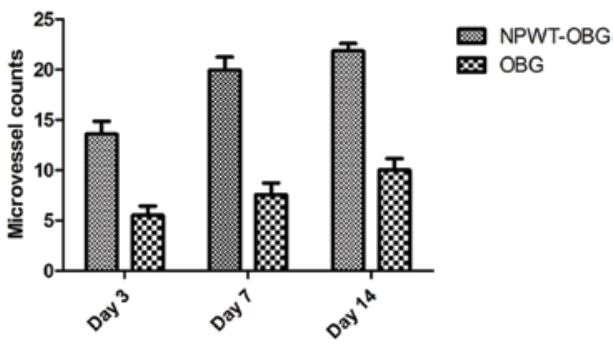

Figure 4. Microvascular density in the granulation tissues was revealed by staining for CD34. (A, C and E) Microvascular density on days 3, 7 and 14, respectively, in the NPWT-OBG group. (B, D and F) Microvascular density on days 3,7 and 14, respectively, in the OBG group (magnification, $\mathrm{x} 400$ ). (G) The number of blood vessels at different times for both groups. NPWT-OBG, negative pressure wound therapy-open bone graft. 

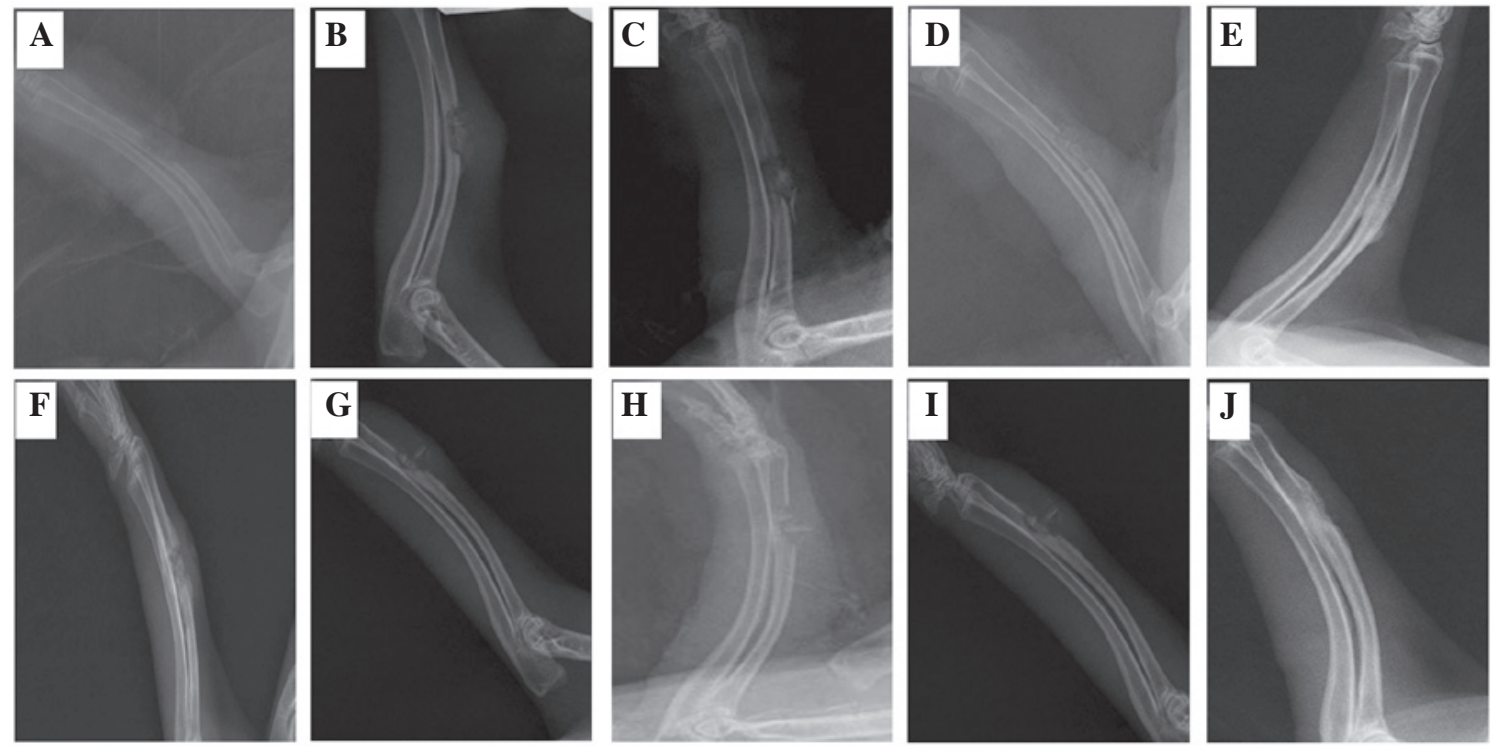

Figure 5. X-ray imaging of the forearm of rabbits at various times. (A-E) X-ray images of the radius on days $0,7,14,21$ and 28 , respectively, in the NPWT-OBG group. (F-J) X-ray images of the radius on days $0,7,14,21$ and 28 , respectively, in the OBG group. NPWT-OBG, negative pressure wound therapy-open bone graft.

OBG has long been used for the treatment of bone defects Papineau et al (9) were the first to report on the application of open cancellous bone grafting in patients with bone defects. However, for bone defects with soft tissue defects, single open bone grafts only repair the bone defect temporarily. As the grafted bone is not covered by soft tissue, the procedure is deemed to be a failure. It indicates that the healing process of soft tissue is important. With this method, the wound was debrided and covered with a conventional dressing. The skin graft was not performed until granulation tissue had filled the wound bed. When the wound had completely healed, the cancellous bone was transplanted. Although the method was simple, the process was too long (10). Thereafter, tissue flap transplantation was developed, which shortened the overall treatment time (2). Unfortunately, the surgery was difficult to master and high-risk.

The NPWT technique, first applied clinically by Fleischmann et al (11) in 1993, is now widely used in surgical drainage, even for complicated wounds. The characteristics that promote wound healing by NPWT are well recognized. Using the NPWT technique, the wound was sealed with a membrane to promote healing. All drawbacks mentioned previously have been solved. Therefore, we combined OBG with NPWT in order to assess whether it was feasible for the treatment of composite bone and soft tissue defects.

Due to the diversity of injuries, wounds are often in contact with the environment without being covered. As a result, the majority of wounds are contaminated with dirt, dust and other pollutants. The majority of open wounds were considered clinically to be polluted wounds. Thus in this study, we investigated polluted wounds in an animal model.

We have identified few relevant reports regarding the use of polluted wound models. Generally, a polluted wound was defined as a wound, open for $<6 \mathrm{~h}$, not severely polluted and without any signs of infection following debridement (12). Thus, we smeared Staphylococcus aureus at a concentration of $1 \times 10^{3}$ cells $/ \mathrm{ml}$ on wounds for 1,2 and $3 \mathrm{~h}$. We found that in open wounds debrided after $1 \mathrm{~h}$, bacteria counting was positive but not infective. This confirmed that the polluted wound model had been successfully established.

It has been recognized that NPWT promotes wound healing. The mechanism been studied thoroughly, with the main focus on the following issues: i) Reducing the edema of tissue surrounding the wound (13); ii) mechanical traction and stimulation with negative pressure; iii) improving local microcirculation in the wound (14); iv) keeping the wound clean in order to inhibit bacterial growth (15); and v) enhancing the expression of relevant factors associated with wound repair $(16,17)$. The first two mechanisms are subjective and not easily measured. Thus we mainly focused on the latter three. In this study, we observed that the bacteria count was reduced in the NPWT-OBG group, but this decrease was not statistically significant when compared with the control group. In the NPWT-OBG group, signs of infection were observed in one rabbit and in three of the rabbits in the OBG group. We suspect that this was due to the small sample size and that if the sample size was increased, greater differences may emerge. Compared with the control group, the number of microvessels increased and VEGF expression was upregulated in the NPWT-OBG group. Increased VEGF typically resulted in increasingly rapid wound closure and facilitated bone healing (18). Thus the combined method has an advantage over conventional therapy for managing wounds with bone and soft tissue defects, and this is in accordance with other studies (19).

Due to its relatively large specific surface area, cancellous bone has a greater number of connections with surrounding tissues (20). Thus, cancellous bone $<0.5 \mathrm{~cm}$ can survive easily. During the surgical procedure, we followed this principle for bone grafting. NPWT research mainly focuses on wound healing, while reports on the effect of NPWT on fracture healing do not exist. Under negative pressure, blood flow is accelerated and the blood supply is increased (21). 
The anti-infection ability was enhanced. All of these factors facilitated the healing process of bone, in accordance with our results.

Though NPWT-OBG exhibited improved results for composite bone and soft tissue defects in rabbits, this may not produce the same result in humans. Composite bone and soft tissue defects in humans are more complicated than those found in animal models and the negative pressure varies. Thus, further research is required.

In conclusion, NPWT may protect polluted wounds from infection. By promoting wound healing, controlling infection and facilitating fracture healing, NPWT-OBG is a feasible treatment for composite bone and soft tissue defects and is more efficient than an OBG. However, whether this remains true for human patients requires further clinical trials.

\section{Acknowledgements}

This project was supported by the National Natural Science Funds of China (grant no. 81171713) and the National Natural Science Funds of Hubei Province (grant no. 2012FFB04311).

\section{References}

1. Yazar S, Lin CH and Wei FC: One-stage reconstruction of composite bone and soft-tissue defects in traumatic lower extremities. Plast Reconstr Surg 114: 1457-1466, 2004.

2. Yu AX, Yu GR, Deng K, Tao SX, Pan ZY and Zhang JH: Combination of negative pressure wound therapy with tissue flap transplantation for severe infectious bone exposure. Chin J Microsurg 29: 219-220, 2006 (In Chinese).

3. Cabanela ME: Open cancellous bone grafting of infected bone defects. Orthop Clin North Am 15: 427-440, 1984.

4. Lei H and Yi L: One-stage open cancellous bone grafting of infected fracture and nonunion. J Orthop Sci 3: 318-323, 1998.

5. Streubel PN, Stinner DJ and Obremskey WT: Use of negative-pressure wound therapy in orthopaedic trauma. J Am Acad Orthop Surg 20: 564-574, 2012.

6. Chen SZ, Li J, Li XY and Xu LS: Effects of vacuum-assisted closure on wound microcirculation: an experimental study. Asian J Surg 28: 211-217, 2005.

7. Vasconcelos MG, Alves PM, Vasconcelos RG, da Silveira EJ, Medeiros AM and de Queiroz LM: Expression of CD34 and CD105 as markers for angiogenesis in oral vascular malformations and pyogenic granulomas. Eur Arch Otorhinolaryngol 268: 1213-1217, 2011.
8. Barrientos S, Stojadinovic O, Golinko MS, Brem H and Tomic-Canic M: Growth factors and cytokines in wound healing. Wound Repair Regen 16: 585-601, 2008.

9. Papineau IJ, Alfageme A, Delcourt JP and Pilon BL: Chronic osteomyelitis of long bones - Resection and bone grafting with delayed skin closure. J Bone Joint Surg (Br) 58: 138-141, 1976.

10. Freeland AE and Mutz SB: Posterior bone-grafting for infected ununited fracture of the tibia. J Bone Joint Surg Am 58: 653-657, 1976.

11. Fleischmann W, Strecker W, Bombelli M and Kinzl L: Vacuum sealing as treatment of soft tissue damage in open fractures. Unfallchirurg 96: 488-492, 1993 (In German).

12. Zhengbao $\mathrm{Z}$ and Youping Y: The clinical significance of preventive use of antibiotics in polluted wound. J Pharm Pract 17: 131-133, 1999.

13. Borgquist $\mathrm{O}$, Ingemansson $\mathrm{R}$ and Malmsjö $\mathrm{M}$ : Wound edge microvascular blood flow during negative-pressure wound therapy: examining the effects of pressures from -10 to -175 mmHg. Plast Reconstr Surg 125: 502-509, 2010.

14. Zhou M, Yu A, Wu G, Xia C, Hu X and Qi B: Role of different negative pressure values in the process of infected wounds healing treated by vacuum-assisted closure: an experimental study. Int Wound J: May 29, 2012 (Epub ahead of print). doi: 10.1111/j.1742-481X.2012.01008.x.

15. Weed T, Ratliff C and Drake DB: Quantifying bacterial bioburden during negative pressure wound therapy: does the wound VAC enhance bacterial clearance? Ann Plast Surg 52: 276-280, 2004.

16. Baldwin C, Potter M, Clayton E, Irvine L and Dye J: Topical negative pressure stimulates endothelial migration and proliferation: a suggested mechanism for improved integration of Integra. Ann Plast Surg 62: 92-96, 2009.

17. Labler L, Rancan M, Mica L, Härter L, Mihic-Probst D and Keel M: Vacuum-assisted closure therapy increases local interleukin-8 and vascular endothelial growth factor levels in traumatic wounds. J Trauma 66: 749-757, 2009.

18. Peng H, Usas A, Olshanski A, et al: VEGF improves, whereas sFlt1 inhibits, BMP2-induced bone formation and bone healing through modulation of angiogenesis. J Bone Miner Res 20: 2017-2027, 2005.

19. Tarkin IS: The versatility of negative pressure wound therapy with reticulated open cell foam for soft tissue management after severe musculoskeletal trauma. J Orthop Trauma 22: S146-S151, 2008.

20. Burchardt H: The biology of bone graft repair. Clin Orthop Relat Res 174: 28-42, 1983.

21. Page JC, Newswander B, Schwenke DC, Hansen M and Ferguson J: Retrospective analysis of negative pressure wound therapy in open foot wounds with significant soft tissue defects. Adv Skin Wound Care 17: 354-364, 2004. 